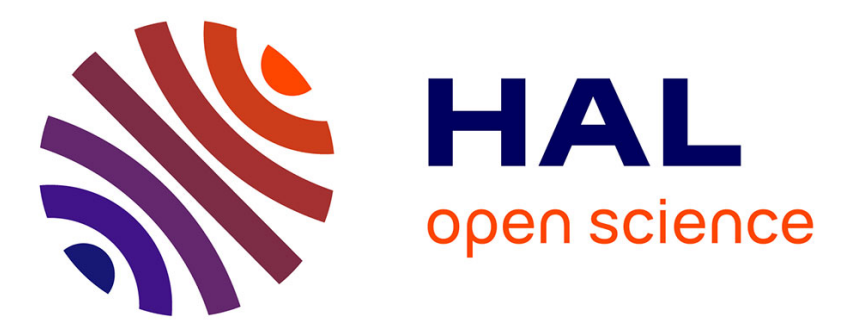

\title{
Direct Observation of the Reduction of a Molecule on Nitrogen Pairs in Doped Graphene
}

\author{
Mehdi Bouatou, Sourav Mondal, Cyril Chacon, Frédéric Joucken, Yann \\ Girard, Vincent Repain, Amandine Bellec, Sylvie Rousset, Shobhana \\ Narasimhan, Robert Sporken, et al.
}

\section{To cite this version:}

Mehdi Bouatou, Sourav Mondal, Cyril Chacon, Frédéric Joucken, Yann Girard, et al.. Direct Observation of the Reduction of a Molecule on Nitrogen Pairs in Doped Graphene. Nano Letters, 2020, 20 (9), pp.6908-6913. 10.1021/acs.nanolett.0c03030 . hal-02992001

\section{HAL Id: hal-02992001 https://hal.science/hal-02992001}

Submitted on 6 Nov 2020

HAL is a multi-disciplinary open access archive for the deposit and dissemination of scientific research documents, whether they are published or not. The documents may come from teaching and research institutions in France or abroad, or from public or private research centers.
L'archive ouverte pluridisciplinaire $\mathbf{H A L}$, est destinée au dépôt et à la diffusion de documents scientifiques de niveau recherche, publiés ou non, émanant des établissements d'enseignement et de recherche français ou étrangers, des laboratoires publics ou privés. 


\title{
Direct observation of the reduction of a molecule on nitrogen pairs in doped graphene
}

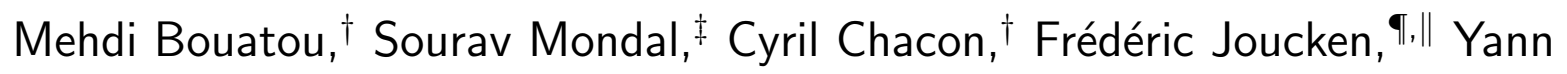 \\ Girard,$^{\dagger}$ Vincent Repain, ${ }^{\dagger}$ Amandine Bellec, ${ }^{\dagger}$ Sylvie Rousset, ${ }^{\dagger}$ Shobhana \\ Narasimhan, ${ }^{\ddagger}$ Robert Sporken, ${ }^{\Uparrow}$ Yannick J. Dappe, ${ }^{\S}$ and Jérôme Lagoute ${ }^{*, \dagger}$ \\ †Université de Paris, Laboratoire Matériaux et Phénomènes Quantiques, CNRS, F-75013, \\ Paris, France \\ $\ddagger$ Theoretical Sciences Unit and School of Advanced Materials, Jawaharlal Nehru Centre for \\ Advanced Scientific Research, Jakkur, Bangalore 560064, India \\ IResearch Center in Physics of Matter and Radiation (PMR), Université de Namur, 61 \\ Rue de Bruxelles, 5000 Namur, Belgium \\ $\S S P E C, C E A, C N R S$, Université Paris-Saclay, CEA Saclay, 91191 Gif-sur-Yvette Cedex, \\ France \\ ||Present address: Department of Physics, University of California, Santa Cruz, California \\ 95060, USA \\ E-mail: jerome.lagoute@univ-paris-diderot.fr
}




\begin{abstract}
Incorporating functional atomic sites in graphene is essential for realizing advanced two-dimensional materials. Doping graphene with nitrogen offers the opportunity to tune its chemical activity, with significant charge redistribution occurring between molecules and substrate. The necessary atomic scale understanding of how this depends on the spatial distribution of dopants, as well as their positions relative to the molecule, can be provided by scanning tunneling microscopy. Here we show that a non-covalently bonded molecule such as $\mathrm{CoPc}$ undergoes a variable charge transfer when placed on N-doped graphene: on a nitrogen pair, it undergoes a redox reaction, with an integral charge transfer, whereas a lower fractional charge transfer occurs over a single nitrogen. Thus the charge state of molecules can be tuned by suitably tailoring the conformation of dopant atoms.
\end{abstract}

\title{
Keywords
}

Scanning tunneling microscopy, Doped graphene, Molecule, Charge transfer

\section{Introduction}

Integrating active atomic sites in graphene is a prerequisite for the realization of second generation two-dimensional materials. Particular attention has been focused on the doping of graphene by heteroatoms, due to the emergence of new physical and chemical properties at well defined point defects. ${ }^{1-6}$ In nitrogen-doped graphene, the doping sites have different electronic properties depending on their atomic structure ${ }^{7}$ and the interaction of molecules with nitrogen sites induces a shift or renormalization of their electronic spectrum. ${ }^{8,9}$ However, redox reactions between doped graphene and molecules have not yet been directly visualized at the molecular scale, and therefore the site dependence of such reactions remains unknown. There is special interest in testing whether one can change the degree of 
oxidation of adsorbed molecules containing a cobalt center binding to four nitrogens $\left(\mathrm{Co}-\mathrm{N}_{4}\right)$, such as cobalt porphyrin $(\mathrm{CoP})$, cobalt phthalocyanine $(\mathrm{CoPc})$ and their derivatives. These molecules have been identified as efficient catalysts for the $\mathrm{CO}_{2}$ reduction reaction. ${ }^{10-12}$ Controlling the charge of these molecules is crucial, since an increase of electron density at the cobalt center amplifies the nucleophilicity, and hence improves its catalytic performance. This effect has been seen on functionalized carbon electrodes where the molecules bind to nitrogenated groups such as pyridine. ${ }^{10,13-15}$ Therefore, the control of the degree of oxidation of the Co- $\mathrm{N}_{4}$ moiety on doped graphene is promising, but remains a challenging task. An atomic scale understanding and control of the charge transfer is still missing, and is highly desirable.

Here we directly observe redox reactions and various degrees of charge transfer between $\mathrm{CoPc}$ molecules and nitrogen-doped graphene, and we unveil the role of the adsorption site on the charge transfer. We show that the charge transfer varies from a fractional to an integer electron charge when a molecule is adsorbed on a single nitrogen atom or a nitrogen pair. Although previous studies have focused on single nitrogen or nitrogen-vacancy combinations, we show that nitrogen pairs can also play a vital role, and that they constitute a reduction center in doped graphene. An intermediate case is also observed, for a molecule centered on a single nitrogen dopant or moved around with respect to the position of a nitrogen pair on the graphene substrate, where the the Lowest Unoccupied Molecular Orbital (LUMO) splits into a singly occupied molecular orbital (SOMO) and a singly unoccupied molecular orbital (SUMO).

\section{Results and discussion}

The system that we study is represented in Fig. 1a. It consists of CoPc molecules on nitrogendoped graphene, where the molecules can sit on a pristine graphene location, single nitrogen site or nitrogen pairs - the latter two are the main nitrogen configurations in our samples. 
The nitrogen pairs consist of two nitrogen atoms separated by a few carbon atoms, ${ }^{7}$ with a $\mathrm{N}-\mathrm{N}$ distance ranging from 2.5 to $10 \AA$. During the deposition process, the diffusion at room temperature leads to the formation of molecular clusters (Fig. 1b) that always include at least one molecule binding to two nitrogen atoms, suggesting that nitrogen pairs act as trapping sites. To locate the underlying nitrogen atoms, the molecules were removed with the STM tip in order to visualize the nitrogen atoms below. The topographs in Fig. 1c,d, taken at $1 \mathrm{~V}$ and $-1.5 \mathrm{~V}$ respectively, show an inversion of the contrast between a CoPc adsorbed on a single nitrogen atom and a $\mathrm{CoPc}$ adsorbed on a nitrogen pair (the same area after spectroscopy measurements and removing the molecules reveals the position of the nitrogen atoms, as seen in Fig. 1e). Note that at higher positive bias (2 V), this contrast disappears, as is shown in Fig. 1b. The nitrogen pair corresponds to a 1-5 pair, with nitrogen atoms separated by two carbon atoms, according to our previous work. ${ }^{7}$ At $-1.5 \mathrm{~V}$, the $\mathrm{CoPc}$ adsorbed on single nitrogen appears with a lobe at the center of the molecule and four double lobes located at the end of the four arms of the molecule (only three are seen due to an overlap of the image of the neighboring molecule). For the molecule on a nitrogen pair, only a single broad lobe is observed on each arm. These contrasts at negative bias are typical of neutral $\mathrm{CoPc}^{0}$ and charged $\mathrm{CoPc}^{-}$molecules. ${ }^{16,17}$ (The molecule is considered to be in a 'neutral' state when only a minute charge transfer occurs, while it is considered to be 'charged' when the charge transfer is close to one electron.) The spectrum of $\mathrm{CoPc}^{0}$ shows resonant states corresponding to a LUMO localized on the Co center, and Highest Occupied Molecular Orbital (HOMO) and LUMO+1 localized on the arms, as evidenced by the $\mathrm{d} I / \mathrm{d} V$ spectra measured at the center and at the arms of the molecule (Fig. 1f). A close look at the spectra shows extra peaks close to the HOMO and LUMO (a shoulder on the right side of the LUMO peak at about $0.43 \mathrm{~V}$ and a small peak below the HOMO state around -1.65 V) that have also been observed in previous studies. ${ }^{16,17}$ The interaction between the CoPc and single nitrogen atom induces a downshift of the molecular spectrum due to a fractional charge transfer, which is similar to previous observations with other molecules ${ }^{8,18,19}$ (see 
supplementary information Fig. S1). When the molecule is bound to two nitrogen atoms, the spectrum, which is shown in Fig. 1g, exhibits a series of peaks at negative bias, typical of a $\mathrm{CoPc}^{-}$molecule, ${ }^{17}$ that are absent on $\mathrm{CoPc}^{0}$. This spectroscopy in the occupied states region can be analyzed as a series of pairs of peaks with the same localization corresponding to different states with vibrational replica. Four states at negative bias emerge: namely, S0 at $-0.20 \mathrm{~V}, \mathrm{~S} 1$ at $-0.29 \mathrm{~V}, \mathrm{~S} 2$ at $-0.76 \mathrm{~V}, \mathrm{~S} 3$ at $-1.0 \mathrm{~V}$ and $\mathrm{S} 4$ at $-1.49 \mathrm{~V}$. The $\mathrm{d} I / \mathrm{d} V$ maps taken at the energies of the peaks of neutral and charged molecules are shown in Fig. 1h. On $\mathrm{CoPc}^{-}$, the first state below the Fermi level, S0, is localised on the cobalt atom, like the LUMO of a neutral molecule, which confirms that the molecule is charged by an electron. The states S1 and S2 are localised on the four arms of the molecule, with one lobe on each arm for S1, and a double lobe on each arm for S2. Each of these states is accompanied by a replica at an energy separation between $0.1 \mathrm{~V}$ and $0.2 \mathrm{~V}$, as seen in Fig. $1 \mathrm{~g}$,h, attributed to vibronic transitions (marked by the index v). At $-0.39 \mathrm{~V}$ and $-1.0 \mathrm{~V}$, a mixing of a state and a vibrational replica appear, due to energy proximity of these features. This distribution of occupied states with vibronic replicas, and the localisation of the states, correspond to the spectrum and conductance maps of $\mathrm{CoPc}^{-}$previously reported on another substrate, ${ }^{17}$ which confirms the assignment of the spectroscopic features that we measure on $\mathrm{CoPc}^{-}$. In the unoccupied region, the conductance map of $\mathrm{CoPc}^{-}$shows a broad feature, related to the broad peak around $1.2 \mathrm{~V}$ (less intense than the LUMO+1 of the neutral molecule) localized on the four arms of the molecule.

The observation of neutral and charged molecules raises the question of the strength of the molecule-surface binding when a molecule is charged or neutral. In order to gain some insight into this problem, we have investigated the possibility of observing a double tunnel barrier effect that is indicative of a weak interaction. ${ }^{19,20}$ In Fig. 2a, we show a cluster

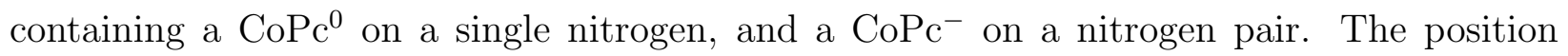
of the nitrogen atoms is revealed in Fig. $2 \mathrm{~b}$ after removing the molecules. The differential conductance curves of $\mathrm{CoPc}^{0}$ (Fig. 2c) and $\mathrm{CoPc}^{-}$(Fig. 2d) exhibit two new peaks at 

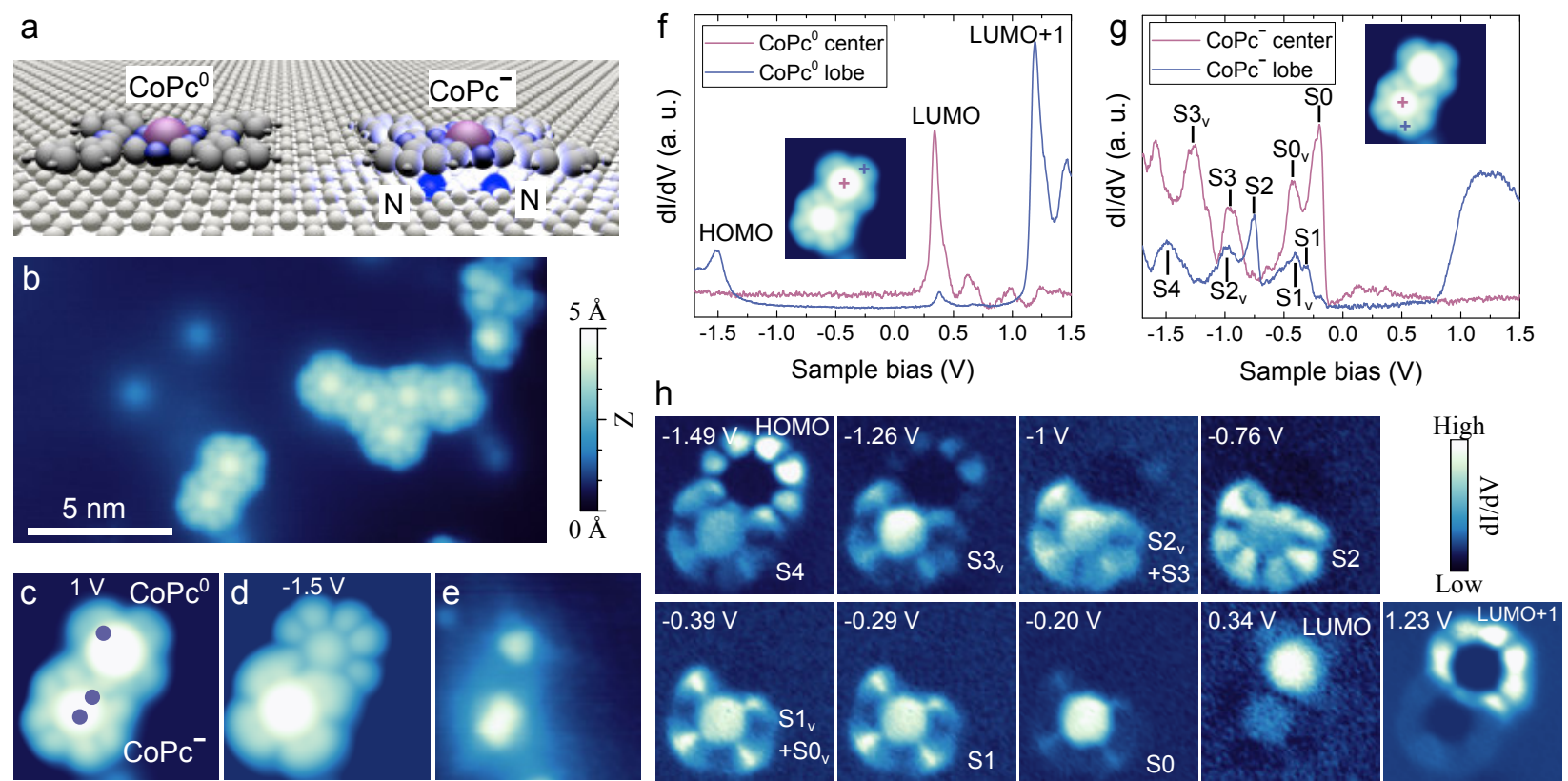

Figure 1: (a) Schematic view of a nitrogen-doped graphene sample with CoPc adsorbed in the neutral state on a pristine site, and negatively charged on a nitrogen pair site. (b) STM image $(2 \mathrm{~V}, 10 \mathrm{pA})$ of CoPc molecules adsorbed on nitrogen-doped graphene. (c,d) Zoomedin view of two molecules from image (b) measured at $1 \mathrm{~V}$ (c) and $-1.5 \mathrm{~V}$ (d) (tunneling current $50 \mathrm{pA}$ ). The dark blue dots in (c) indicate the location of nitrogen atoms below the molecules. Image sizes $4 \mathrm{~nm} \times 4 \mathrm{~nm}$. (e) Image of the same area after removing the molecules, revealing the nitrogen atoms below the molecules $(0.5 \mathrm{~V}, 100 \mathrm{pA})$. The $\mathrm{d} I / \mathrm{d} V$ spectra of the two molecules of $(c, d)$ are shown in $(f, g)$. The insets in $(f, g)$ indicate the locations where the spectra were measured. (h) Conductance maps of the same area as (c,d) at sample bias voltages corresponding to the peaks observed in $(\mathrm{f}, \mathrm{g})$. 
negative (around $-1.9 \mathrm{~V}$ ) and positive (around $0.3 \mathrm{~V}$ ) bias respectively, that have a different physical origin as compared to the resonant states. These peaks shift significantly upon changing the tip height, which is typical of a charging peak. ${ }^{19}$ For a weakly bound molecule, the potential drop of the tunneling junction is distributed between the tip-molecule barrier and the molecule-substrate area, behaving as a second tunneling barrier. Therefore, upon the application of a bias voltage, the molecular levels are shifted, and when a molecular state crosses the Fermi level of the sample, it gains or loses one electron, leading to a new charge state of the molecule. Such a charging effect has been seen on various systems where the molecule-surface interaction is weak or when a molecule is adsorbed on a metal covered by an insulating film. ${ }^{19,21-26}$ In Fig. 2, these peaks appear when the LUMO of a neutral molecule (Fig. 2c) or $\mathrm{LUMO}^{-}$of a charged molecule (Fig. 2d) is shifted towards the Fermi level, leading to a temporary charging of the molecule. The charging peaks are not observed in the cases presented in Fig. 1, because they are outside the voltage range used in the spectroscopic measurements. For the neutral molecule in Fig. 1, the LUMO is at a higher energy $(0.34 \mathrm{~V})$ than in Fig.2, therefore its charging peak is expected to occur at a larger negative bias (below -1.9 V). The situation is similar for the charged molecule in Fig. 1 with a $\mathrm{LUMO}^{-}$at a more negative bias $(-0.2 \mathrm{~V})$ than in Fig.2 (-0.08 V), therefore its charging peak is expected at a large bias voltage.

It has to be noted that the peaks corresponding to the resonant states are also slightly shifted when the tip height changes, though to a much lesser extent. To unambiguously identify the origin of the different peaks, we use a double tunneling barrier junction model in order to estimate the effect of the electric field. ${ }^{27}$ In a double barrier, the condition for a resonant tunneling with a molecular level aligned with the Fermi level of the tip is obtained when $e V_{b}=E_{0}(1+d / \epsilon z)$, where $V_{b}$ is the sample bias voltage, $E_{0}$ is the energy of the states, $z$ the distance between tip and sample, $d$ the distance between the molecule and the surface and $\epsilon$ is the dielectric constant of the area between the molecule and the surface. For the charging peak, the condition to align the molecular level with the Fermi level of the sample is 

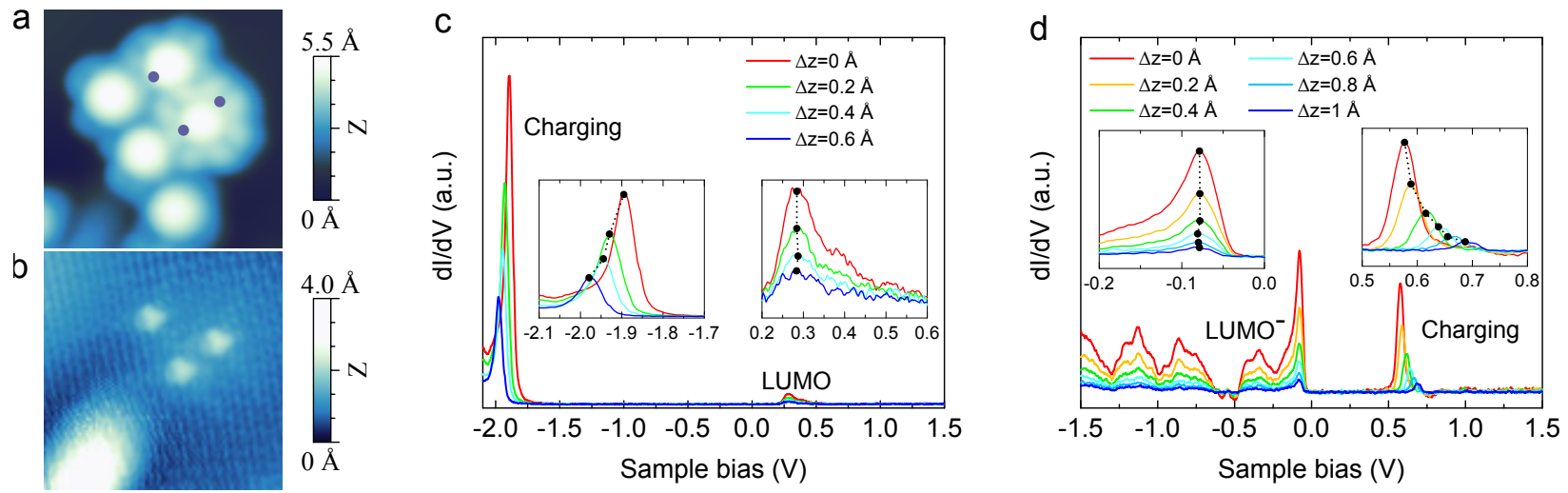

Figure 2: (a) STM image (1V, $50 \mathrm{pA})$ of a CoPc cluster including a molecule adsorbed on a nitrogen pair and a molecule on a single nitrogen site. The dark blue dots indicate the position of the nitrogen atoms below the molecules. Image size $5 \mathrm{~nm} \times 5 \mathrm{~nm}$. (b) STM image $(0.2 \mathrm{~V}, 300 \mathrm{pA})$ of the same area as in (a), after removing the molecules, revealing the location of nitrogen atoms. (c,d) $\mathrm{d} I / \mathrm{d} V$ spectra measured at the center (above the Co atom) of the molecule shown in panel (a) that is (c) adsorbed on a single nitrogen and (d) on the nitrogen pair, for different tip heights. The tip-sample separation was increased by the amount $\Delta z$ from the setpoint $1 \mathrm{~V}, 50 \mathrm{pA}$. The insets are zoomed-in views of the LUMO and charging peaks.

$e V_{b}=-E_{0}(1+\epsilon z / d)$. It is important to note that these relations allow for an unambiguous determination of the origin of a peak, from its variation with the tip height. When the tip-sample distance increases, the energy of the peak is shifted away from the Fermi level for a charging peak, whereas it moves towards the Fermi level for a resonant molecular state. Moreover, for a given change in the tip-sample separation, the shift of a resonant peak is much smaller than the shift of a charging peak. From this model, the variation of the charging peaks of $\mathrm{CoPc}^{0}$ and $\mathrm{CoPc}^{-}$can be reproduced, together with a nearly absent shift for the resonant states (see supplementary information Fig. S2). As a result, the peak at positive bias measured at the center of the charged molecule can unambiguously be identified as a charging peak and not a LUMO state. The double barrier behaviour observed on the charged molecule shows that the molecule-graphene interface can be modeled as a tunneling barrier, indicative of a weak coupling between the molecule and the substrate. Note that in the case of $\mathrm{CoPc}$ on hexagonal boron nitride $(\mathrm{h}-\mathrm{BN})$ on $\operatorname{Ir}(111)$ the charging peaks were not observed. ${ }^{17}$ Another difference is that on $\mathrm{h}-\mathrm{BN}$ the charging of $\mathrm{CoPc}$ involves the supporting 
substrate, while here the reduction of $\mathrm{CoPc}$ is induced by the material itself and its dopants.
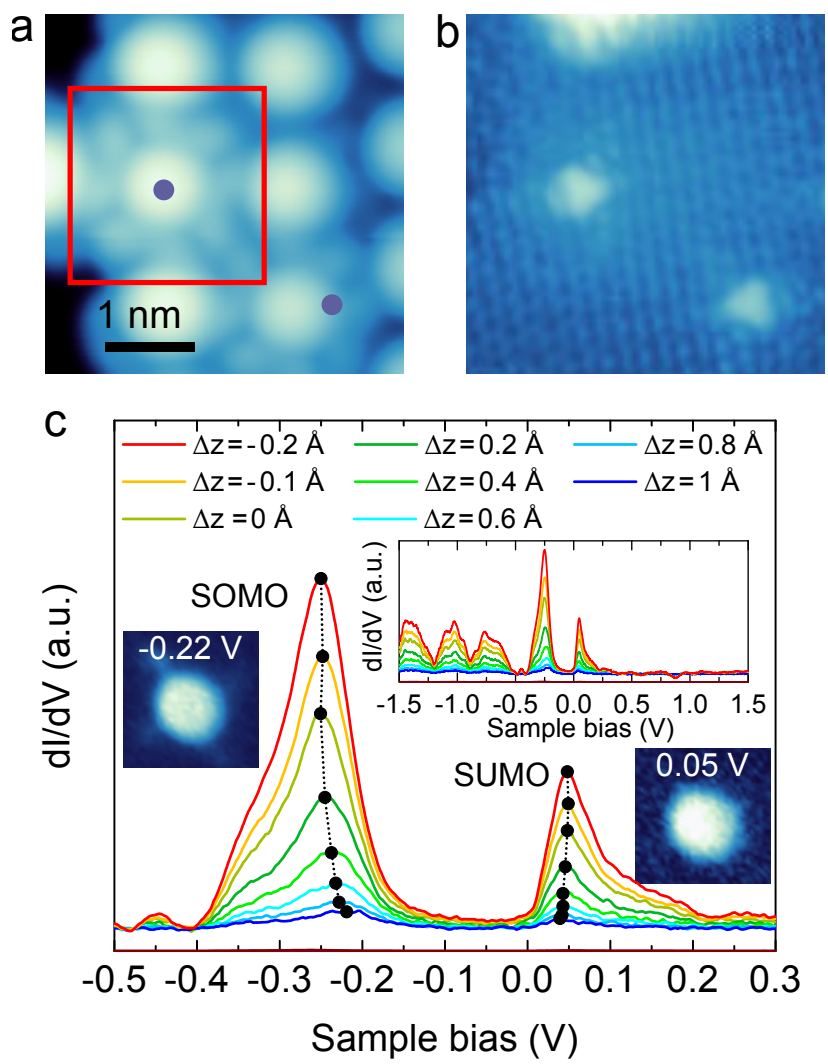

Figure 3: (a) STM image (1V, $50 \mathrm{pA}$ ) of CoPc molecules including a molecule (marked by a red square) adsorbed above a nitrogen atom located directly below the Co atom. The dark blue dots indicate the position of the nitrogen atoms below the molecules. (b) STM image $(0.2 \mathrm{~V}, 300 \mathrm{pA})$ of the same area as in (a), after removing the molecules, revealing the location of nitrogen atoms. (c) $\mathrm{d} I / \mathrm{d} V$ spectra measured at the center of the molecule marked by a square in (a). The tip-sample separation was changed by the amount $\Delta z$ from the setpoint position at $1 \mathrm{~V}, 50 \mathrm{pA}$. Negative values of $\Delta z$ correspond to a decrease of the tip-sample separation. The marks, linked by dotted lines, indicate the peak position of the SOMO and SUMO states. The inset curves show the spectra for a larger voltage range. The images in the two square insets correspond to the $\mathrm{d} I / \mathrm{d} V$ maps in the area marked by a square in (a) at -0.22 and $0.05 \mathrm{~V}$.

We have seen that nitrogen pairs lead to a stronger charge transfer than single nitrogen atoms. An intermediate case between the neutral and charged molecules described above is obtained when a molecule is adsorbed above a single nitrogen atom located at the center of the molecule. In that case, the $\mathrm{d} I / \mathrm{d} V$ spectrum shows a series of peaks at negative bias, typical of a charged molecule, and also exhibits two peaks at positive and negative bias close to the Fermi level (Fig. 3), corresponding to a split LUMO state, as we will discuss below. 
In contrast to the case shown in Fig. 2d, these two peaks shift towards the Fermi level when the tip is retracted, showing that no charging peak is present. This feature is typical of a half-filled molecular state that splits into two levels separated by a Coulomb repulsion energy, leading to the formation of a singly occupied molecular orbital (SOMO) and a singly unoccupied molecular orbital (SUMO). The $\mathrm{d} I / \mathrm{d} V$ maps measured on the molecule at the energies of the SOMO and SUMO states reveal that these states are localised on the center of the molecule (inset in Fig. 3c). This shape is characteristic of the LUMO of a neutral molecule, which confirms that the two peaks around the Fermi level correspond to SOMO and SUMO states. The surrounding molecules do not show this feature, but instead exhibit the expected spectrum of a neutral molecule, more or less shifted depending on the amount of charge transfer (see supplementary information Fig. S3). The existence of SOMO and SUMO states could also be observed for a molecule adsorbed on a nitrogen pair that could be switched between neutral and charged states by lateral manipulation with the STM tip. In its charged state, the molecule showed a SOMO and SUMO state feature (see supplementary information Fig. S4-S5).

Our experimental data show that nitrogen atoms allow for charge transfer to noncovalently bonded molecules on graphene, and more particularly they show that nitrogen pairs induce an integer electron charge transfer. This is further supported by our Density Functional Theory (DFT) calculations. We present in Fig. 4a,b the spin-polarized Projected Density of States (PDOS) of the CoPc molecules on graphene and on a 1-5 nitrogen pair in N-doped graphene corresponding to the case of Fig. 1 (see also supplementary information Fig. S6). Since DFT is a ground state theory, it is well-known that HOMO-LUMO gaps are computed incorrectly, and one cannot therefore expect to obtain exact agreement between the PDOS computed theoretically, and the $\mathrm{d} I / \mathrm{d} V$ spectrum obtained experimentally. For example, a standard feature of DFT computations of metal-phthalocyanines on substrates 
is that the LUMO peak appears at the Fermi level, in disagreement with experimental observations. Similarly, absolute values for charges on atoms, as computed from DFT, can be unreliable, due to ambiguities in charge-partitioning schemes. However, DFT results for lowest-energy geometries and trends in charge transfer are accurate enough to provide considerable physical insight. In particular, DFT can reliably state when the charge transfer is larger or smaller, which is of interest to us here. When comparing the two PDOS computed on pristine and N-doped graphene, we can clearly observe a shift of the LUMO level below the Fermi level in the latter case, in good agreement with the experimental observations. This shift corresponds to a charging of the molecule from the nitrogen atoms doped in graphene. Indeed, the corresponding charge transfers computed from DFT are 0.20 e from graphene to $\mathrm{CoPc}$ on pristine graphene, and 0.61 e on $\mathrm{N}$-doped graphene. Our calculations also reveal an inverse relationship between the Co-substrate distance and the charge transfer (see supplementary information Fig. S7). The addition of the electron charge also reduces the magnetic moment from $0.76 \mu_{B}$ on pristine graphene to $0.54 \mu_{B}$ on $\mathrm{N}$-doped graphene. The charge transfer and the shift of the PDOS is found to be smaller on single nitrogen than on a nitrogen pair (see supplementary information Fig. S8-S9). Due to the DFT limitations discussed before, the calculated charge transfer does not correspond exactly to that deduced from the experiments, but, importantly, follows the same trend, and reproduces well the shift of the LUMO level below the Fermi level. Finally, when considering the isosurfaces of the charge density redistribution, shown in Fig. 4c,d, we find that the charge transfer is enhanced when a pair of nitrogen atoms is present in the graphene below the $\mathrm{CoPc}$ molecule. This is obviously due to the specific location of the molecule above these atoms, as represented in Fig. 4e.

In conclusion, we have shown that nitrogen pairs in doped graphene allow one to reduce cobalt phthalocyanine molecules. An integral charge transfer toward the molecules is evidenced by the shift of the LUMO state below the Fermi level. In some particular cases, when the molecule is sitting on a single nitrogen atom or a nitrogen pair, the downshifted peak 

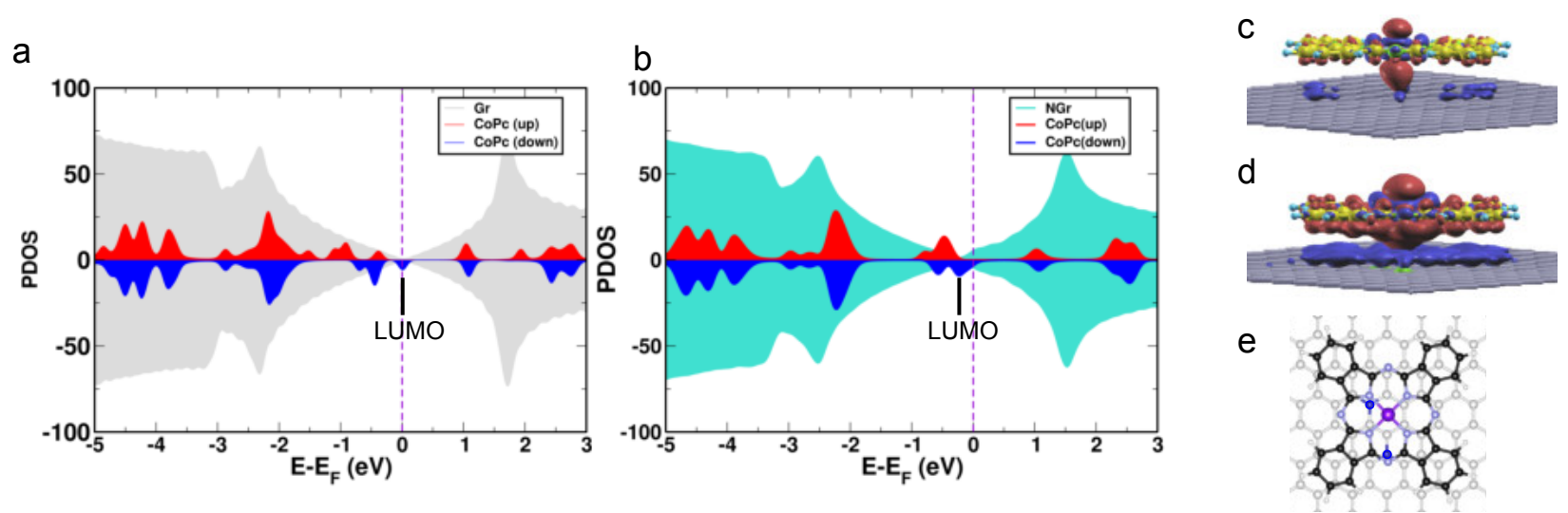

Figure 4: (a) PDOS computed from DFT, of graphene (gray) and CoPc (spin-up in red, spindown in blue), for CoPc adsorbed on pristine graphene. (b) PDOS of graphene (light blue) and $\mathrm{CoPc}$ (spin-up in red, spin-down in blue), for CoPc adsorbed on doped graphene with two nitrogen atoms below the molecule. (c-d) Isosurfaces $\left(0.0005 \mathrm{e} / \mathrm{bohr}^{3}\right)$ of the charge density redistribution for (c) CoPc on pristine graphene and (d) $\mathrm{CoPc}$ on a nitrogen pair in doped graphene. Red/blue lobes correspond to electron accumulation/depletion, respectively. (e) Top view of the structure used for the calculations of $\mathrm{CoPc}$ on a nitrogen pair in doped graphene. Color code: black for carbon in $\mathrm{CoPc}$, gray for carbon in graphene, light blue for nitrogen in $\mathrm{CoPc}$, dark blue for nitrogen in graphene, white for hydrogen, purple for Co.

gets split into a SUMO and SOMO. This effect on the molecules could have potential applications in improving the catalytic activity of $\mathrm{CoPc}$, for reactions such as the reduction of $\mathrm{CO}_{2}$ for energy conversion. More generally, nitrogen-doped graphene can be used to control the charge state of molecules on graphene.

\section{Acknowledgement}

This project has received funding from the European Union's Horizon 2020 research and innovation program under grant agreement No. 766726. We acknowledge funding from CEFIPRA/IFCPAR.

\section{Supporting Information Available}

Computational details, STM measurements, Shift of molecular spectrum on nitrogen atoms, 
Double tunnel barrier model for charging peaks and resonant states, Spectroscopy of molecules around nitrogen atoms in a cluster, Manipulation of molecules on nitrogen pairs, Geometries used for DFT calculations, Comparison of DFT results for charge transfer, DFT Results for PDOS for CoPc over single $\mathrm{N}$ dopant in graphene.

\section{References}

(1) Joucken, F.; Tison, Y.; Lagoute, J.; Dumont, J.; Cabosart, D.; Zheng, B.; Repain, V.; Chacon, C.; Girard, Y.; Botello-Méndez, A. R.; Others, Localized state and charge transfer in nitrogen-doped graphene. Phys. Rev. B 2012, 85, 161408.

(2) Lv, R.; Terrones, M. Towards new graphene materials: doped graphene sheets and nanoribbons. Mater. Lett. 2012, 78, 209-218.

(3) Wang, H.; Maiyalagan, T.; Wang, X. Review on recent progress in nitrogen-doped graphene: synthesis, characterization, and its potential applications. ACS Catal. 2012, 2, 781-794.

(4) Wang, X.; Sun, G.; Routh, P.; Kim, D.-H.; Huang, W.; Chen, P. Heteroatom-doped graphene materials: syntheses, properties and applications. Chem. Soc. Rev. 2014, 43, 7067-7098.

(5) Putri, L. K.; Ong, W.-J.; Chang, W. S.; Chai, S.-P. Heteroatom doped graphene in photocatalysis: a review. Appl. Surf. Sci. 2015, 358, 2-14.

(6) Joucken, F.; Henrard, L.; Lagoute, J. Electronic properties of chemically doped graphene. Phys. Rev. Mater. 2019, 3, 110301.

(7) Tison, Y.; Lagoute, J.; Repain, V.; Chacon, C.; Girard, Y.; Rousset, S.; Joucken, F.; Sharma, D.; Henrard, L.; Amara, H. , et al. Electronic interaction between nitrogen atoms in doped graphene. ACS Nano 2015, 9, 670-678. 
(8) Pham, V. D.; Lagoute, J.; Mouhoub, O.; Joucken, F.; Repain, V.; Chacon, C.; Bellec, A.; Girard, Y.; Rousset, S. Electronic interaction between nitrogen-doped graphene and porphyrin molecules. ACS Nano 2014, 8, 9403-9409.

(9) de la Torre, B.; Švec, M.; Hapala, P.; Redondo, J.; Krejčí, O.; Lo, R.; Manna, D.; Sarmah, A.; Nachtigallová, D.; Tuček, J.; Błoński, P.; Otyepka, M.; Zboil, R.; Hobza, P.; Jelínek, P. Non-covalent control of spin-state in metal-organic complex by positioning on N-doped graphene. Nat. Commun. 2018, 9, 1-9.

(10) Manbeck, G. F.; Fujita, E. A review of iron and cobalt porphyrins, phthalocyanines and related complexes for electrochemical and photochemical reduction of carbon dioxide. J. Porphyr. and Phthalocya. 2015, 19, 45-64.

(11) Zhang, Z.; Xiao, J.; Chen, X.-J.; Yu, S.; Yu, L.; Si, R.; Wang, Y.; Wang, S.; Meng, X.; Wang, Y. , et al. Reaction Mechanisms of Well-Defined Metal-N4 Sites in Electrocatalytic CO2 Reduction. Angew. Chem. Int. Ed. 2018, 57, 16339-16342.

(12) Nam, D.-H.; De Luna, P.; Rosas-Hernández, A.; Thevenon, A.; Li, F.; Agapie, T.; Peters, J. C.; Shekhah, O.; Eddaoudi, M.; Sargent, E. H. Molecular enhancement of heterogeneous CO 2 reduction. Nat. Mater. 2020, 19, 266-276.

(13) Atoguchi, T.; Aramata, A.; Kazusaka, A.; Enyo, M. Electrocatalytic activity of CoII TPP-pyridine complex modified carbon electrode for CO2 reduction. J. Electroanal. Chem. 1991, 318, 309-320.

(14) Tanaka, H.; Aramata, A. Aminopyridyl cation radical method for bridging between metal complex and glassy carbon: cobalt (II) tetraphenylporphyrin bonded on glassy carbon for enhancement of CO2 electroreduction. J. Electroanal. Chem. 1997, 437, $29-35$.

(15) Zhu, M.; Cao, C.; Chen, J.; Sun, Y.; Ye, R.; Xu, J.; Han, Y. F. Electronic Tuning of 
Cobalt Porphyrins Immobilized on Nitrogen-Doped Graphene for CO2 Reduction. ACS Appl. Energy Mater. 2019, 2, 2435-2440.

(16) Schulz, F.; Drost, R.; Hamalainen, S. K.; Liljeroth, P. Templated self-assembly and local doping of molecules on epitaxial hexagonal boron nitride. ACS Nano 2013, 7, $11121-11128$.

(17) Schulz, F.; Ijäs, M.; Drost, R.; Hämäläinen, S. K.; Harju, A.; Seitsonen, A. P.; Liljeroth, P. Many-body transitions in a single molecule visualized by scanning tunnelling microscopy. Nat. Phys. 2015, 11, 229-234.

(18) Pham, V. D.; Joucken, F.; Repain, V.; Chacon, C.; Bellec, A.; Girard, Y.; Rousset, S.; Sporken, R.; dos Santos, M. C.; Lagoute, J. Molecular adsorbates as probes of the local properties of doped graphene. Sci. Rep. 2016, 6, 24796.

(19) Pham, V. D.; Ghosh, S.; Joucken, F.; Pelaez-Fernandez, M.; Repain, V.; Chacon, C.; Bellec, A.; Girard, Y.; Sporken, R.; Rousset, S.; Dappe, Y. J.; Narasimhan, S.; Lagoute, J. Selective control of molecule charge state on graphene using tip-induced electric field and nitrogen doping. npj 2D Mater. Appl. 2019, 3, 1-5.

(20) Wickenburg, S. et al. Tuning charge and correlation effects for a single molecule on a graphene device. Nat. Commun. 2016, 7, 1-7.

(21) Pradhan, N. A.; Liu, N.; Silien, C.; Ho, W. Atomic Scale Conductance Induced by Single Impurity Charging. Phys. Rev. Lett. 2005, 94, 076801.

(22) Teichmann, K.; Wenderoth, M.; Loth, S.; Ulbrich, R. G.; Garleff, J. K.; Wijnheijmer, A. P.; Koenraad, P. M. Controlled Charge Switching on a Single Donor with a Scanning Tunneling Microscope. Phys. Rev. Lett. 2008, 101, 076103.

(23) Brar, V. W.; Decker, R.; Solowan, H.-M.; Wang, Y.; Maserati, L.; Chan, K. T.; Lee, H.; Girit, C. O.; Zettl, A.; Louie, S. G.; Cohen, M. L.; Crommie, M. F. Gate-controlled 
ionization and screening of cobalt adatoms on a graphene surface. Nat. Phys. 2011, 7, $43-47$.

(24) Wang, Y.; Brar, V. W.; Shytov, A. V.; Wu, Q.; Regan, W.; Tsai, H.-Z.; Zettl, A.; Levitov, L. S.; Crommie, M. F. Mapping Dirac quasiparticles near a single Coulomb impurity on graphene. Nat. Phys. 2012, 8, 653-657.

(25) Fernández-Torrente, I.; Kreikemeyer-Lorenzo, D.; Stróżecka, A.; Franke, K. J.; Pascual, J. I. Gating the Charge State of Single Molecules by Local Electric Fields. Phys. Rev. Lett. 2012, 108, 036801.

(26) Kocic, N.; Weiderer, P.; Keller, S.; Decurtins, S.; Liu, S.-X.; Repp, J. Periodic Charging of Individual Molecules Coupled to the Motion of an Atomic Force Microscopy Tip. Nano Lett. 2015, 15, 4406-4411.

(27) Wu, S.; Nazin, G.; Chen, X.; Qiu, X.; Ho, W. Control of relative tunneling rates in single molecule bipolar electron transport. Phys. Rev. Lett. 2004, 93, 236802 


\section{Graphical TOC Entry}

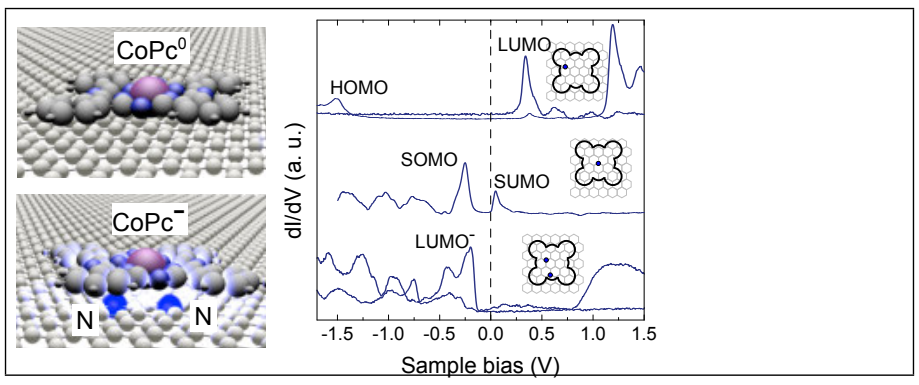

